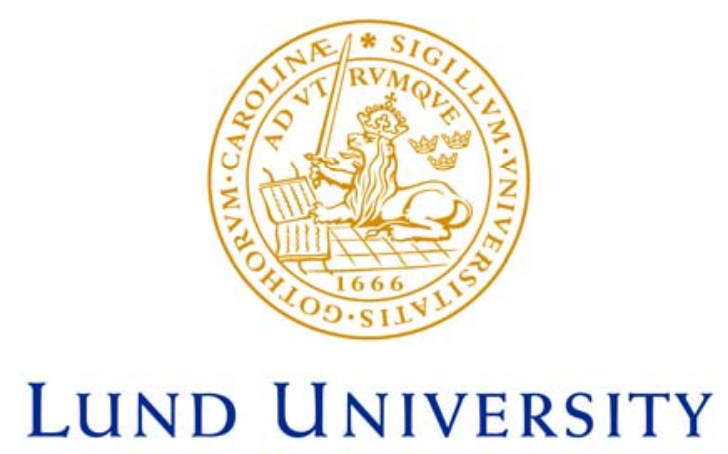

Faculty of Medicine

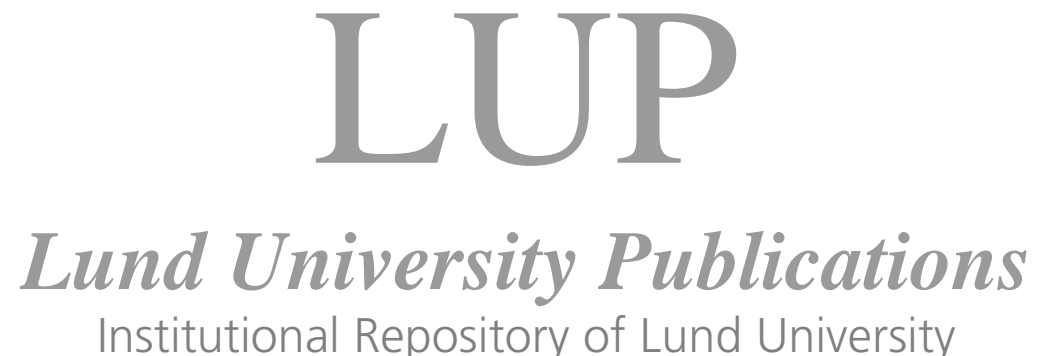

Institutional Repository of Lund University

This is an author produced version of a paper published in Trends in immunology. This paper has been peer-reviewed but does not include the final publisher proof-corrections or journal pagination.

Citation for the published paper:

Kutty Selva Nandakumar, Rikard Holmdahl.

"Therapeutic cleavage of IgG: new avenues for treating inflammation. "

Trends in immunology, 2008, Issue: March 5.

http://dx.doi.org/10.1016/j.it.2008.01.007

Access to the published version may require journal subscription.

Published with permission from: Elsevier 


\section{Therapeutic cleavage of IgG - new avenues for treating inflammation}

Kutty Selva Nandakumar* and Rikard Holmdahl

Medical Inflammation Research, Lund University, Lund and Karolinska Institutet, Stockholm, Sweden.

*Corresponding author: Nandakumar, K.S. (nan@med.lu.se)

\section{Contact Details:}

1. Kutty Selva Nandakumar, Ph.D., Dr.Med.Sci.

Medical Inflammation Research,

I-11, BMC, Lund University

Sweden.

and

Medical Inflammation Research,

MBB, Karolinska Institutet

Sweden.

Phone: +46-46-222 3349; Fax: +46-46-222 3110; e-mail: nan@med.lu.se 
2. Rikard Holmdahl, M.D., Ph.D.

Medical Inflammation Research,

I-11, BMC, Lund University

Sweden

and

Medical Inflammation Research,

MBB, Karolinska Institutet

Sweden.

Phone:+46-46-2224607;Fax:+46-46-2223110;e-mail: rikard.holmdahl@,med.lu.se 


\section{Abstract}

Autoantibodies developing in humans contribute to the pathogenesis of several diseases and injected therapeutic antibodies can also trigger adverse side effects. An efficient and rapid elimination of these antibodies are therefore critically needed. Antibody removal by plasmapheresis and immunoadsorption are commonly used methods but have their own limitations. Bacterial enzymes that can cleave IgG molecules or remove carbohydrate moieties to ameliorate their immunogenicity or effector functions in vivo offer new avenues for drug development. Recent discoveries highlight the possibility of cleaving or modifying IgG in vivo by injection of enzymes. The possibility to modify IgG structures in vivo or ex vivo opens up new therapeutic possibilities not only for pathogenic antibody-mediated inflammatory diseases but also harmful effects caused by transplantations or treatment with "biologicals". 


\section{Rationale}

B cells antibodies and immune complexes are implicated in the pathogenesis of various inflammatory diseases. Similarly, alloantibodies have been shown to contribute to both early and late graft loss [1] and antibodies are increasingly used as therapeutics. Hence treatments targeting antibodies, present new possibilities for the treatment of inflammatory disorders such as rheumatoid arthritis (RA), systemic lupus erythematosus (SLE), myasthenia gravis (MG), idiopathic thrombocytopenic purpura (ITP), hemophilia, pemphigus vulgaris (PV), Grave's disease (GD) and organ-allograft rejection.

\section{B cell depletion therapy}

The importance of B cells in autoimmune diseases has been well documented [2-4]. B cells contribute to the disease process as antigen presenting cells, through costimulatory functions (surface molecules, secreted cytokines and interaction with chemokines) as well as by secreting pathogenic antibodies. Recent success in B cell depletion therapies especially using anti-CD20 antibodies (rituximab) in autoimmune diseases for example RA and SLE is encouraging [5-8]. However, it takes a long time before the antibody levels in serum decreases and an ameliorative effect on disease is seen. Antibodies remains in circulation, probably produced by long-lived plasma cells in the bone marrow [9] where they have to receive survival signals from stromal cells [10]. FcrRIIB was found to be critical for prevention of generation and expansion of autoreactive antibody secreting IgG positive plasma cells [11] and cross-linking this inhibitory receptor on plasma cells in the bone marrow to induce apoptosis has been suggested as a means to reduce the pathogenic antibodies in circulation $[12,13]$. 


\section{Antibody functions and autoimmunity}

The antibody (humoral) response in vertebrates is produced against foreign structures and is intended for the protection of hosts against invading pathogenic microorganisms but excessive activation to overcome self-tolerance or misguided immune activation leads to generation of self-reactive antibodies that are potentially pathogenic. It is clear that the presence of autoantibodies precedes the onset of autoimmune diseases in humans, for example in type 1 diabetes and arthritis [6, 14]. Apart from being diagnostic and prognostic tools in several disease conditions, antibodies have several vital functions in the humoral immune and inflammatory response of the host: interacting with Fc receptors (FcR) and complement components, activation of phagocytes, modulation of B cell functions (memory induction, activation and/or feedback inhibition of antibody production), neutralization of toxins, antibody-dependent cellular cytotoxicity (ADCC), activation of natural killer cells and eosinophils, modulation of dendritic cell function, mast cell degranulation and opsonization of antigens leading to enhanced phagocytosis.

The role of antibodies in autoimmune diseases and in biological treatments is the subject of growing interest and has led to the need for understanding the effector functions of antibodies in vivo. Antibodies as a constituent of immune complexes play a pivotal role in triggering several inflammatory processes. Multimeric complexes formed by antibodies binding to self-antigens can initiate an inflammatory attack by destabilizing the tissue, possibly by release of tissue degrading enzymes and cytokines by the infiltrating cells [15]. The bound antibodies can trigger and enhance inflammation by optimal activation of the complement cascade and/or Fc $\gamma \mathrm{R}$-bearing cells. Complement 
fragments binding to immune complexes, target tissue damage, and Fc $\gamma \mathrm{R}$ cross-linking can activate local mononuclear cells that in turn release proinflammatory cytokines in or near the target tissue inducing neutrophil and macrophage recruitment. These phagocytes can be further activated and create a pro-inflammatory cytokine milieu that can affect the activities of resident cell populations present in the target organs. Release of granules containing many tissue-degrading enzymes and, reactive oxygen and nitrogen radicals by macrophages and neutrophils can amplify their responses thereby forming a vicious cycle damaging the end organs and so cause clinical pathology. Thus, antibodies binding to human self-antigens contribute to the pathogenesis that can lead to severe clinical manifestations.

Antibodies can also directly cause the destruction of their target tissue preceding and independent of disease development and in the absence of any other pathogenic inflammatory factors or the action of immunocytes. In RA, antibodies reactive to type II collagen in the cartilage matrix are directly pathogenic even in the absence of inflammatory mediators [15]. For instance they can impair cartilage formation [16], strongly inhibit collagen fibrillogenesis [17], disrupt collagen fibrils in the extracellular matrix with or without increased matrix synthesis [18], and have deleterious effects on the pre-formed cartilage [19] which all suggest that chondrocyte-reactive antibodies might play an important pathogenic role leading to irreversible cartilage damage in RA. Similarly, IgG autoantibodies from pemphigus patients can exert a direct effect by binding to epidermal cells and this is mediated by steric hindrance and/or by triggering the transduction of a signal to the cell, thereby disrupting structures that maintain cell- 
cell or cell-matrix adhesion in the skin $[20,21]$. These findings showed that the antibodies alone could initiate the pathogenic events even before the inflammatory phase of the disease.

Similarly, neutralizing IgG antibodies present in up to $50 \%$ of hemophilia A patients inactivate and neutralize the therapeutic administration of exogenous factor VIII by sterically hindering its interaction with molecules of the coagulation cascade, or by forming immune complexes leading to accelerated clearance from the circulation [22]. Table 1 shows a list of autoimmune diseases in which $\operatorname{IgG}$ plays a major role in mediating the disease processes. Elimination of such pathogenic IgG from circulation in these autoimmune patients becomes all the more vital for remission of the clinical pathology.

\section{Removal of circulating antibodies and limitations of current methods}

Antibodies as therapeutic targets gained ground with several clinical and pre-clinical (animal models) observations demonstrating the pathogenic capacity of antibodies to induce a variety of autoimmune diseases. Removal of circulating immune complexes and pathogenic antibodies by therapeutic plasmapheresis or immunoadsorption [23] in many autoimmune diseases proved to be beneficial but are expensive, cumbersome for the patients and takes time. Plasmapheresis is limited by its non-selective removal of all plasma components and requires plasma product replacement such as human albumin or fresh frozen plasma. Although immunoadsorption techniques aim to provide more specific elimination of antibodies with fewer side effects compared to plasmapheresis, extensive immunoadsorption is unavoidable. The number of patients treated by 
immunoadsorption also remains small due to a lack of well-defined controlled trials and limited benefits. Moreover the biomaterials used in apheresis, activated complement components and heightened the risk of hemolysis, thrombosis and infections [24, 25]. However, current biocompatible materials (dextran sulfate, protein A - silica or sepharose, tryptophan, phenylalanine, and Ig - Therasorb columns) seem able to avoid these adverse phenomena. Furthermore, it is important to note that antibodies are more or less evenly distributed both in the intra- and the extravascular compartments and inflammatory processes often occur in the tissue but not in the vasculature. Hence, elimination of antibodies from the circulation alone is not sufficient to control the inflammatory processes [26]. Moreover, repeated and prolonged treatments are absolutely needed to avoid redistribution of pathogenic autoantibodies to the target tissues, although concomitant intravenous immunoglobulin injection might mitigate the effect of Ig adsorption. However, complete elimination of immunoglobulin might lead to immunodeficiency resulting in severe opportunistic infections. Hence, studies focused on developing tools for the transient elimination of antibodies are crucial for treating autoantibody-mediated pathologies.

\section{Exploiting bacterial enzymes for the control of autoimmune diseases}

Agents which directly target pathogenic antibodies and so mimic the strategy evolved by infectious microorganisms in trying to avoid the immune system is an interesting therapeutic concept. Diversion of the adaptive immune system by cleaving antibodies is a common mechanism utilized by many pathogenic bacterial species and several microbial extracellular enzymes with immunomodulating activities have been identified [27]. The Gram-positive bacterium Streptococcus pyogenes is a common human pathogen, often 
causing relatively mild clinical conditions such as pharyngitis, scarlet fever and impetigo. However, invasive strains can penetrate into deeper tissues and cause severe or lifethreatening infections such as necrotizing fasciitis, sepsis and streptococcal toxic shock syndrome [28]. The IgG degrading enzyme of S. pyogenes (IdeS) (also designated as Mac1) is a cysteine endopeptidase, secreted by group A streptococcal strains during infection. It cleaves the heavy chains of IgG with a unique specificity by binding and cleaving in the hinge region (Fig. $1 \mathrm{~A}$ and $2 \mathrm{~B}$ ), thus generating an $\mathrm{Fc}$ and an $\mathrm{F}\left(\mathrm{ab}^{\prime}\right) 2$ fragment $[29,30]$ that can be detected by protein G capture and mass spectrometry [31]. By removing the $\mathrm{Fc}$ section from the antigen recognizing Fab, immune responses such as complement deposition, and Fc-mediated phagocytosis are blocked. This IgG proteolytic degradation disables opsonophagocytosis and interferes with the killing of group A Streptococcus [28]. Most likely, IdeS bestows a local protective effect for the bacteria. However, the unique specificity and ability of IdeS to cleave all human IgG [28] in vivo also provides a possibility to target pathogenic antibodies. Most importantly, IdeS has a higher degree of specificity for $\operatorname{IgG}$ than previously described proteinases with proteolytic activity towards immunoglobulins, e.g. papain, pepsin or streptococcal pyrogenic exotoxin B $[29,30]$ In the mouse, only $\operatorname{IgG} 2 \mathrm{a}, \operatorname{IgG} 2 \mathrm{c}$ and $\mathrm{IgG} 3$ are cleaved and injections of IdeS showed a rapid, specific and efficient cleavage of these subclasses of IgG antibodies [32] . In fact, antibodies inducing arthritis could be inhibited in vivo by IdeS treatment (Fig.2A and 2B) in experimental murine models (collagen induced arthritis; CIA and collagen antibody induced arthritis; CAIA) of RA [32]. IdeS treatment reduced the severity of arthritis by cleaving both circulating and cartilage bound antibodies if administered within 24 hours after the onset of clinical arthritis, but did not 
block ongoing severe arthritis. IdeS treatment also significantly prevented antibody induced relapse in mice that had chronic arthritis, and delayed the onset and reduced the severity of arthritis in classic CIA. However, cleavage of BCR by "IgG specific" IdeS has not been clearly ascertained, but it should be noted that early B cell progenitors are IgMexpressing and the resurgence of IgG2a antibody levels after IdeS treatment in CIA [32] do however argue against the action of IdeS on BCR. It should also be mentioned that that this IgG cleaving therapy will not directly affect plasma cells as they do not express BCR. However, in these experiments, all of the $\operatorname{IgG} 2 \mathrm{a} / \mathrm{c}$ and $\mathrm{IgG} 3$ antibodies were cleaved and removed, and also replaced after IdeS had been cleared from the circulation, thereby avoiding the permanent elimination of any potentially protective antibodies. Furthermore, it is also possible that IdeS generated Fab fragments of autoreactive antibodies would still bind to and block their antigens but not elicit an effector response to the target. Similarly, the free Fc fragments could block Fc receptors on phagocytes (similar to 2.4G2) and so could prevent inflammation. Streptokinase, another proteolytic enzyme of S. pyogenes, has been used as a therapeutic (thrombolytic) agent in humans for decades demonstrating the safety of using bacterial enzymes for therapy. Hence, IdeS treatment could be a new strategy to cleave pathogenic IgG.

Yet another strategy, which targets IgG, is by the removal of carbohydrate moieties with bacterial enzymes. Post-translational modifications, especially glycosylation, have important effects on the structure and biological properties of glycoproteins such as immunoglobulins [33-35]. IgG molecules are mainly glycosylated through covalent attachment at Asn-297 of the $\mathrm{CH} 2$ domain within the $\mathrm{Fc}$ region with variable 
galactosylation but limited sialylation. The remainder of the glycosylation occurs in the hypervariable regions of the Fab. These complex biantennary-type oligosaccharides attached to IgG are essential for effector functions mediated through $\mathrm{Fc}$ receptors and complement C1q [36, 37], apart from being critically involved in maintaining the structural integrity of the antibody [38]. X-ray crystallographic studies have revealed multiple non-covalent interactions between the sugar and protein resulting in reciprocal influences on their structural conformation [38, 39]. Modifications in these oligosaccharides affect susceptibility to proteolytic degradation, clearance rate, ADCC and complement dependent cytotoxicity [35, 40]. De-fucosylation on the N297-linked glycan in the Fc part of the antibody increases its binding capacity significantly to activatory Fc $\gamma$ RIV in mice and Fc $\gamma$ RIIIA in humans, and also enhanced ADCC activity suggesting the importance of glyco-engineering of antibodies for improved therapy [4144]. On the other hand, recent studies demonstrated that higher Fc sialylation of IgG leads to its anti-inflammatory $[45,46]$ as well as reduced pathogenic [47] properties apart from decreased ADCC activity [48]. However, exactly how the oligosaccharides influence the Fc structure and function remains to be elucidated.

Recently, a novel secreted endo- $\beta-N$-acetylglucosaminidase, a member of the glycosyl hydrolases of family 18 (FGH18) in S. pyogenes (EndoS), which specifically hydrolyzes the $\beta$-1,4-di- $N$-acetylchitobiose core of the asparagine-linked glycan of human $\operatorname{IgG}$ was identified [27]. EndoS has similarities to endo- $\beta-N$-acetylglucosaminidases that cleave the $\beta 1-4$ linkage between the two $N$-acetylglucosamines found in the core of the $\mathrm{N}$-linked glycan of IgG (Fig.1B and 2C). EndoS exclusively hydrolyzes the complex-type 
biantennary glycan on the heavy $\gamma$-chain of native $\operatorname{IgG}$ [27]. Endoglycosidase activity on the IgG molecule by EndoS alters its function through impaired FcyR binding as well as decreased activation of the classical pathway of complement, which ultimately leads to increased bacterial survival in human blood. Recent results also demonstrated the effect of specific removal of carbohydrate moieties from $\operatorname{IgG}$ by EndoS, which results in reduced binding to Fc $\gamma$ Rs and formation of less stable immune complexes, and therefore inhibiting experimental arthritis induction [49].

\section{Concluding remarks}

The targeted cleavage of pathogenic antibodies with a rapid and efficient destruction of the antibody effector mechanisms would be highly beneficial in several types of disorders and clinical conditions. Patients who have antibodies that are agonistic (e.g. Grave's disease), neutralizing (acquired FVIII deficiency and myasthenia gravis), complement and macrophage activating (Goodpasture's syndrome and autoimmune bullous skin diseases), depleting (autoimmune hemolytic anemia, idiopathic thrombocytopenic purpura) or immune complex associated (RA, systemic lupus erythematosus) could be benefited by treatments targeting elimination of pathogenic $\operatorname{IgG}$. There are also situations created by modern health care in which antibodies may play a pathogenic role. For instance during acute rejection of transplants, antibodies play an important role and there is a need for an immediate reversal of their effect $[1,50,51]$. Likewise, the expanding use of various therapeutic antibodies such as rituximab (anti-CD20) warrants the possibility of immediately blocking their actions in case of unforeseen or severe side effects. A dramatic recent example is the severe adverse effects observed in a phase I clinical trial of an activatory anti-CD28 mAb [52], where the rapid removal of the mAb could have 
ameliorated the symptoms. Interestingly, similar to $\operatorname{IgG}$ cleaving bacterial enzymes, recent studies with proteases from schistosoma parasites demonstrated human IgE-Fc cleavage [53], which presents possibilities for treating IgE-mediated allergic conditions.

Bacterial enzyme(s) that can cleave IgG transiently or involved in selective removal of carbohydrate moieties thus provide new avenues for blocking pathogenic antibodies in vivo. However, antibodies generated against these enzymes could pose problems in treating the patients [54]. Hence, further analyses are required to determine the efficacy of these enzymes in cleaving the substrate in the presence of such neutralizing antibodies. Similarly, construction of fusion proteins with active component of the enzyme that induced negligible amounts of neutralizing antibodies will be useful for clinical applications.

\section{Acknowledgements}

We thank the following foundations for financial support. Professor Nanna Svartz, Alfred

Österlund, King Gustav V 80 years, the Swedish Association against Rheumatism, the Swedish Strategic Research Foundation and the EU projects LSHB-CT-2006-018661 (AUTOCURE) and LSHG-CT-2005-005203 (MUGEN). 


\section{References}

1 Colvin, R. B. and Smith, R. N. (2005) Antibody-mediated organ-allograft rejection. Nat Rev Immunol 5 (10), 807-817

2 Tuscano, J. M. et al. (2003) B lymphocytes contribute to autoimmune disease pathogenesis: current trends and clinical implications. Autoimmun Rev 2 (2), 101108

3 Martin, F. and Chan, A. C. (2006) B cell immunobiology in disease: evolving concepts from the clinic. Annu Rev Immunol 24, 467-496

4 Blank, M. and Shoenfeld, Y. (2007) B cell targeted therapy in autoimmunity. J Autoimmun 28 (2-3), 62-68

5 Edwards, J. C. et al. (2004) Efficacy of B-cell-targeted therapy with rituximab in patients with rheumatoid arthritis. N Engl J Med 350 (25), 2572-2581

6 Browning, J. L. (2006) B cells move to centre stage: novel opportunities for autoimmune disease treatment. Nat Rev Drug Discov 5 (7), 564-576

7 Silverman, G. J. (2007) Anti-CD20 therapy and autoimmune disease: therapeutic opportunities and evolving insights. Front Biosci 12, 2194-2206

8 Sanz, I. et al. (2007) B cell depletion therapy in autoimmune diseases. Front Biosci 12, 2546-2567

9 Manz, R. A. et al. (2005) Maintenance of serum antibody levels. Annu Rev Immunol 23, 367-386

10 Radbruch, A. et al. (2006) Competence and competition: the challenge of becoming a long-lived plasma cell. Nat Rev Immunol 6 (10), 741-750 
11 Fukuyama, H. et al. (2005) The inhibitory Fcgamma receptor modulates autoimmunity by limiting the accumulation of immunoglobulin G+ anti-DNA plasma cells. Nat Immunol 6 (1), 99-106

12 Xiang, Z. et al. (2007) FcgammaRIIb controls bone marrow plasma cell persistence and apoptosis. Nat Immunol 8 (4), 419-429

13 Ravetch, J. V. and Nussenzweig, M. (2007) Killing some to make way for others. Nat Immunol 8 (4), 337-339

14 Scofield, R. H. (2004) Autoantibodies as predictors of disease. Lancet 363 (9420), $1544-1546$

15 Nandakumar, K. S. et al. (2007) Arthritogenic antibodies specific for a major type II collagen triple-helical epitope bind and destabilize cartilage independent of inflammation. Arthritis Rheum 58 (1), 184-196

16 Amirahmadi, S. F. et al. (2004) An arthritogenic monoclonal antibody to type II collagen, CII-C1, impairs cartilage formation by cultured chondrocytes. Immunol Cell Biol 82 (4), 427-434

17 Gray, R. E. et al. (2004) Measurement of antibodies to collagen II by inhibition of collagen fibril formation in vitro. J Immunol Methods 285 (1), 55-61

18 Amirahmadi, S. F. et al. (2005) Arthritogenic anti-type II collagen antibodies are pathogenic for cartilage-derived chondrocytes independent of inflammatory cells. Arthritis Rheum 52 (6), 1897-1906

19 Crombie, D. E. et al. (2005) Destructive effects of murine arthritogenic antibodies to type II collagen on cartilage explants in vitro. Arthritis Res Ther 7 (5), R927937 
20 Sitaru, C. and Zillikens, D. (2005) Mechanisms of blister induction by autoantibodies. Exp Dermatol 14 (12), 861-875

21 Sitaru, C. et al. (2007) The relevance of the IgG subclass of autoantibodies for blister induction in autoimmune bullous skin diseases. Arch Dermatol Res 299 (1), 1-8

22 Lacroix-Desmazes, S. et al. (2006) Catalytic IgG from patients with hemophilia A inactivate therapeutic factor VIII. J Immunol 177 (2), 1355-1363

23 Stegmayr, B. G. (2005) A survey of blood purification techniques. Transfus Apher Sci 32 (2), 209-220

24 Gorbet, M. B. and Sefton, M. V. (2004) Biomaterial-associated thrombosis: roles of coagulation factors, complement, platelets and leukocytes. Biomaterials 25 (26), 5681-5703

25 Nilsson, B. et al. (2007) The role of complement in biomaterial-induced inflammation. Mol Immunol 44 (1-3), 82-94

26 Gaubitz, M. and Schneider, K. M. (2003) Immunoadsorption in systemic lupus erythematosus: different techniques and their current role in medical therapy. Ther Apher Dial 7 (2), 183-188

27 Collin, M. and Olsen, A. (2003) Extracellular enzymes with immunomodulating activities: variations on a theme in Streptococcus pyogenes. Infect Immun 71 (6), 2983-2992

28 von Pawel-Rammingen, U. and Bjorck, L. (2003) IdeS and SpeB: immunoglobulin-degrading cysteine proteinases of Streptococcus pyogenes. Curr Opin Microbiol 6 (1), 50-55 
29 Vincents, B. et al. (2004) Enzymatic characterization of the streptococcal endopeptidase, IdeS, reveals that it is a cysteine protease with strict specificity for IgG cleavage due to exosite binding. Biochemistry 43 (49), 15540-15549

30 Wenig, K. et al. (2004) Structure of the streptococcal endopeptidase IdeS, a cysteine proteinase with strict specificity for IgG. Proc Natl Acad Sci U S A 101 (50), 17371-17376

31 Hess, J. L. et al. (2007) Immunoglobulin cleavage by the streptococcal cysteine protease IdeS can be detected using protein G capture and mass spectrometry. J Microbiol Methods 70 (2), 284-291

32 Nandakumar, K. S. et al. (2007) Blocking of experimental arthritis by cleavage of IgG antibodies in vivo. Arthritis Rheum 56 (10), 3253-3260

33 Jefferis, R. (2005) Glycosylation of recombinant antibody therapeutics. Biotechnol Prog 21 (1), 11-16

34 Walsh, G. and Jefferis, R. (2006) Post-translational modifications in the context of therapeutic proteins. Nat Biotechnol 24 (10), 1241-1252

35 Arnold, J. N. et al. (2007) The impact of glycosylation on the biological function and structure of human immunoglobulins. Annu Rev Immunol 25, 21-50

36 Nezlin, R. and Ghetie, V. (2004) Interactions of immunoglobulins outside the antigen-combining site. Adv Immunol 82, 155-215

37 Nimmerjahn, F. and Ravetch, J. V. (2007) Fc-Receptors as Regulators of Immunity. Adv Immunol 96C, 179-204 
38 Krapp, S. et al. (2003) Structural analysis of human IgG-Fc glycoforms reveals a correlation between glycosylation and structural integrity. J Mol Biol 325 (5), $979-989$

39 Jefferis, R. (2006) A sugar switch for anti-inflammatory antibodies. Nat Biotechnol 24 (10), 1230-1231

40 Kanda, Y. et al. (2007) Comparison of biological activity among nonfucosylated therapeutic IgG1 antibodies with three different N-linked Fc oligosaccharides: the high-mannose, hybrid, and complex types. Glycobiology 17 (1), 104-118

41 Shinkawa, T. et al. (2003) The absence of fucose but not the presence of galactose or bisecting $\mathrm{N}$-acetylglucosamine of human IgG1 complex-type oligosaccharides shows the critical role of enhancing antibody-dependent cellular cytotoxicity. J Biol Chem 278 (5), 3466-3473

42 Nimmerjahn, F. and Ravetch, J. V. (2005) Divergent immunoglobulin g subclass activity through selective Fc receptor binding. Science 310 (5753), 1510-1512

43 Nechansky, A. et al. (2007) Compensation of endogenous IgG mediated inhibition of antibody-dependent cellular cytotoxicity by glyco-engineering of therapeutic antibodies. Mol Immunol 44 (7), 1815-1817

44 Jefferis, R. (2007) Antibody therapeutics: isotype and glycoform selection. Expert Opin Biol Ther 7 (9), 1401-1413

45 Kaneko, Y. et al. (2006) Anti-inflammatory activity of immunoglobulin G resulting from Fc sialylation. Science 313 (5787), 670-673

46 Nimmerjahn, F. and Ravetch, J. V. (2007) The antiinflammatory activity of IgG: the intravenous IgG paradox. J Exp Med 204 (1), 11-15 
47 Baudino, L. et al. (2006) Molecular and cellular basis for pathogenicity of autoantibodies: lessons from murine monoclonal autoantibodies. Springer Semin Immunopathol 28 (2), 175-184

48 Scallon, B. J. et al. (2007) Higher levels of sialylated Fc glycans in immunoglobulin G molecules can adversely impact functionality. Mol Immunol $44(7), 1524-1534$

49 Nandakumar, K. S. et al. (2007) Endoglycosidase treatment abrogates IgG arthritogenicity: Importance of IgG glycosylation in arthritis. Eur J Immunol 37 (10), 2973-2982

50 Cai, J. and Terasaki, P. I. (2005) Humoral theory of transplantation: mechanism, prevention, and treatment. Hum Immunol 66 (4), 334-342

51 Terasaki, P. and Mizutani, K. (2006) Antibody mediated rejection: update 2006. Clin J Am Soc Nephrol 1 (3), 400-403

52 Sheridan, C. (2006) TeGenero fiasco prompts regulatory rethink. Nat Biotechnol $24(5), 475-476$

53 Aslam, A. et al. (2008) Proteases from Schistosoma mansoni cercariae cleave IgE at solvent exposed interdomain regions. Mol Immunol 45 (2), 567-574

54 Akesson, P. et al. (2006) IdeS, a highly specific immunoglobulin G (IgG)cleaving enzyme from Streptococcus pyogenes, is inhibited by specific IgG antibodies generated during infection. Infect Immun 74 (1), 497-503

55 Gearing, A. J. et al. (2002) Selective cleavage of human IgG by the matrix metalloproteinases, matrilysin and stromelysin. Immunol Lett 81 (1), $41-48$ 


\section{Figure legends}

Figure 1: IdeS and EndoS cleavage sites on IgG molecule. (A) Amino acid sequence of the constant region of human IgG1 heavy chain with IdeS cleaving site. Other major protease cleavage sites are also shown for comparison. (B) The oligosaccharide attached to asparagine 297 in the antibody $\mathrm{Fc}$ fragment is of the complex biantennary type. EndoS hydrolyses the chitobiose core of the glycan. After EndoS activity, innermost N-acetyl glycosamine is still left attached with Asn-297 with a core fucose moiety. Cleavage sites for peptide-N-glycosidase F (PNGase F) and neuraminidase are also shown. GlcNAc, $N$ acetylglucosamine; Fuc, fucose; Man, mannose; Gal, galactose; NeuAc, sialic acid (source $[27,30,55])$.

Figure 2: Effect of IdeS and EndoS cleavage on IgG and experimental arthritis (CAIA). (A) Untreated IgG antibodies induced arthritis in naïve mice, (B) IdeS treatment cleaved IgG into an $\mathrm{Fc}$ and an $\mathrm{F}\left(\mathrm{ab}^{\prime}\right) 2$ fragment leading to blocking of arthritis induction and (C) EndoS treatment cleaved carbohydrate moieties after fucose in the $\mathrm{CH} 2$ domain of $\operatorname{IgG}$ abrogating IgG arthritogenicity. CHO, carbohydrate. Representative pictures of mouse ankle joint sections stained with hematoxylin and eosin. Original magnification x 10 . (source $[32,49])$. 
Table1: Diseases with IgG mediated pathology

\begin{tabular}{|c|c|c|}
\hline Autoimmune disease & Clinical condition & Target (s) \\
\hline $\begin{array}{l}\text { Graves' or Basedows' } \\
\text { disease }\end{array}$ & Hyperthroidism & Thyroid gland (TSHR) \\
\hline Acquired FVIII deficiency & Hemophilia & Factor VIII \\
\hline Myasthenia gravis & $\begin{array}{l}\text { Muscle weakness and } \\
\text { fatiguability }\end{array}$ & Skeletal muscle (AchR) \\
\hline Goodpasture's syndrome & $\begin{array}{l}\text { Glomerulonephritis, } \\
\text { Pneumonitis }\end{array}$ & $\begin{array}{l}\text { Lund, Kidney (Collagen } \\
\text { type IV) }\end{array}$ \\
\hline $\begin{array}{l}\text { Autoimmune hemolytic } \\
\text { anemia }\end{array}$ & cytopenia & Red blood cells \\
\hline $\begin{array}{l}\text { Idiopathic } \\
\text { thrombocytopenia }\end{array}$ & cytopenia & Platelets \\
\hline Autoimmune neutropenias & cytopenia & Neutrophils \\
\hline Mixed cryoglobulinemia & Pupura, Nephritis, Neuritis & $\begin{array}{l}\text { Sometimes associated with } \\
\text { hepatitis } \mathrm{C} \text { infection }\end{array}$ \\
\hline $\begin{array}{l}\text { Systemic lupus } \\
\text { erythematosus }\end{array}$ & $\begin{array}{l}\text { Discoid lupus, Nephritis, } \\
\text { Anemia }\end{array}$ & Systemic (dsDNA, ssDNA) \\
\hline ANCA associated vasculitis & $\begin{array}{l}\text { Purpura, arthritis, } \\
\text { Hemoptysis }\end{array}$ & Vasculature \\
\hline Rheumatoid arthritis & $\begin{array}{l}\text { Inflammation in articular } \\
\text { joints }\end{array}$ & Joints \\
\hline Sjögren's syndrome & $\begin{array}{l}\text { Inflammation in lacrimal } \\
\text { glands }\end{array}$ & $\begin{array}{l}\text { Salivary gland. RNP } \\
\text { proteins Ro (SSA), La } \\
\text { (SSB) }\end{array}$ \\
\hline Antiphospholipid syndrome & Blood clotting disorder & $\begin{array}{l}\text { Vasculature (coagulation } \\
\text { factors) }\end{array}$ \\
\hline Pemphigus diseases & Bullous skin diseases & $\begin{array}{l}\text { Skin, Mucous membranes, } \\
\text { Keratinocytes }\end{array}$ \\
\hline $\begin{array}{l}\text { Complete congenital heart } \\
\text { block }\end{array}$ & Cardiovascular disease & $\begin{array}{l}\text { RNP proteins Ro (SSA), La } \\
\text { (SSB) }\end{array}$ \\
\hline $\begin{array}{l}\text { Primary progressive } \\
\text { Multiple Sclerosis }\end{array}$ & Neurological disorder & $\begin{array}{l}\text { Myelin oligodendrocyte } \\
\text { glycoprotein }\end{array}$ \\
\hline Chronic Urticaria & $\begin{array}{l}\text { Cutaneous mast cell } \\
\text { degranulation }\end{array}$ & $\begin{array}{l}\text { Alpha-subunit of the IgE } \\
\text { receptor }\end{array}$ \\
\hline
\end{tabular}

TSHR-Thyroid stimulating hormone receptor; AchR-Acetyl choline receptor; ANCAAnti-neutrophil cytoplasmic antibodies; RNP- Ribonuclear proteins 

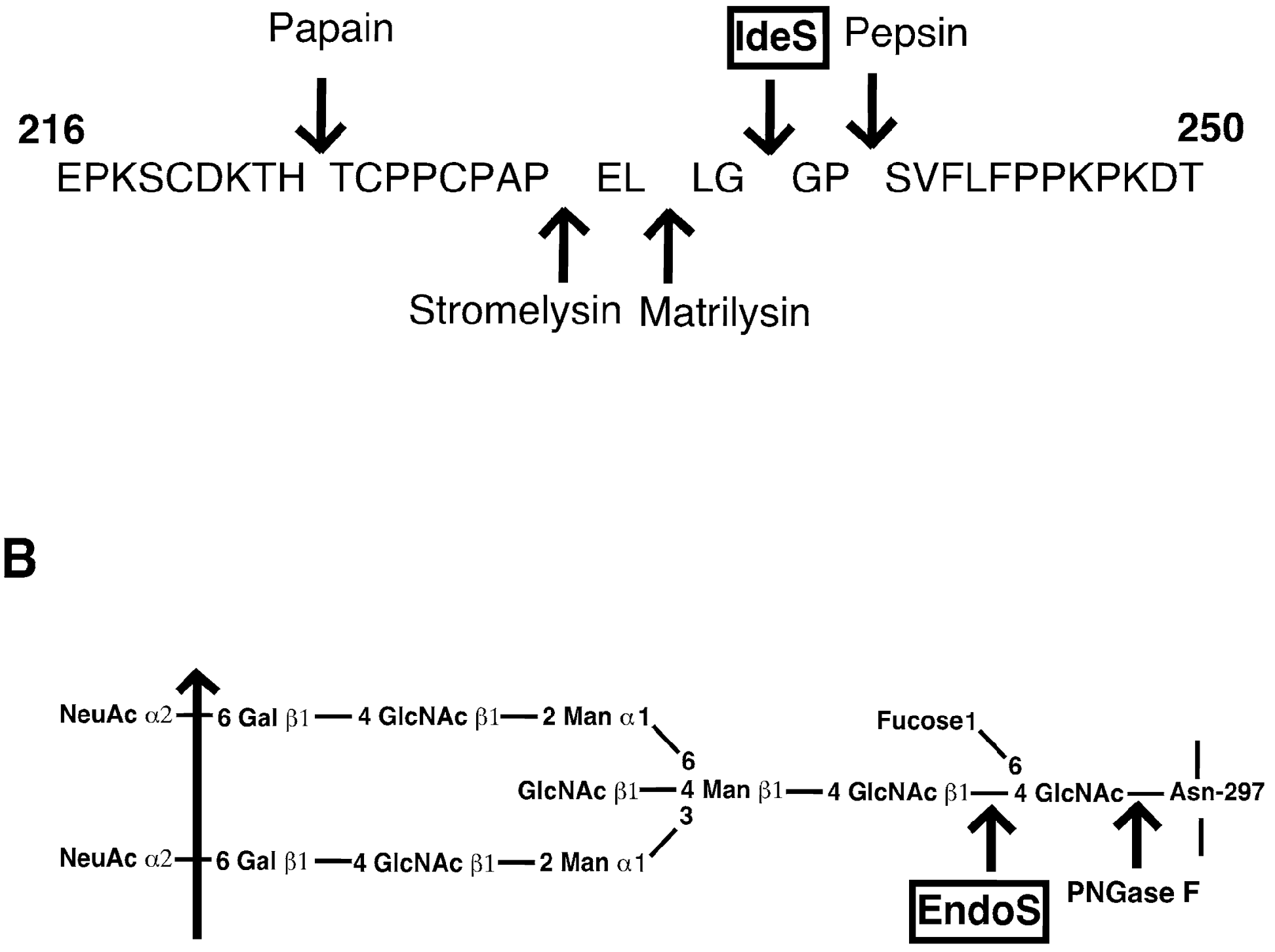

Neuraminidase 
